\title{
Effect of the cumulative dose of zoledronic acid on the pathogenesis of osteonecrosis of the jaws
}

\author{
MERAL GÜNALDI $^{1}$, CIGDEM USUL AFSAR ${ }^{2}$, BERNA BOZKURT DUMAN ${ }^{3}$, \\ ISMAIL OGUZ KARA $^{2}$, UFUK TATLI ${ }^{4}$ and BERKSOY SAHIN ${ }^{2}$ \\ ${ }^{1}$ Department of Medical Oncology, Bakirkoy Dr Sadi Konuk Training and Research Hospital, Istanbul; \\ ${ }^{2}$ Department of Medical Oncology, Cukurova University Medical School; ${ }^{3}$ Department of Medical Oncology, \\ Adana Research and Training Hospital; ${ }^{4}$ Faculty of Dentistry, Cukurova University, Adana, Adana, Turkey
}

Received July 10, 2014; Accepted March 24, 2015

DOI: $10.3892 / \mathrm{ol} .2015 .3156$

\begin{abstract}
Bisphosphonate-related osteonecrosis of the jaws (BRONJ) is a severe bone disease for which the pathogenetic mechanisms and risk factors are not fully understood. The present study evaluated the data of 652 patients with bone metastasis that had undergone treatment with biphosphonates. Subsequently, 24 patients with BRONJ and 20 control patients without BRONJ that were treated with zoledronic acid were enrolled. It was found that BRONJ occurred in $3.6 \%$ of patients. The mean age and the administration of dental treatment were found to be significantly associated with BRONJ development $(\mathrm{P}=0.049$ and $\mathrm{P}=0.013$, respectively). The cumulative dose median in the BRONJ group was found to be significantly higher compared with the cumulative dose average in the control group $(\mathrm{P}=0.037)$. In addition, at the time of BRONJ development, improvement in the disease was determined to be better in the BRONJ group than in the control group $(\mathrm{P}=0.031)$. The present study determined that age, the existence of dental extraction and the cumulative dose of zoledronate were all important risk factors in BRONJ development.
\end{abstract}

\section{Introduction}

Bisphosphonates (BPs) are synthetic drugs used in the treatment of osteolytic bone disorders, including osteoporosis, Paget's disease, bone metastasis and multiple myeloma (MM) $(1,2)$. BPs are pyrophosphate analogues with a high affinity for hydroxyapatite crystals $(3,4)$. Once bound to hydroxyapatite crystals, BPs are slowly released during bone resorption and are subsequently internalised by osteoclasts. BPs inhibit osteoclastic bone resorption by interfering with

Correspondence to: Dr Meral Günaldi, Department of Medical Oncology, Bakirkoy Dr Sadi Konuk Training and Research Hospital, 11 Tevfik Sağlam Street, Bakirkoy, Istanbul 34147, Turkey E-mail: meralgunaldi@gmail.com

Key words: zoledronic acid, cumulative dose, jaw, osteonecrosis, risk factors osteoclast recruitment, differentiation and activity, and by promoting apoptosis (5-8). In addition, BPs are reported to exert direct antiproliferative and proapoptotic effects on cancer cells, thereby reducing bone metastases $(9,10)$. Furthermore, BPs alter angiogenesis (11) and signal transduction between osteoclasts and osteoblasts (12). Depending on the presence or absence of a nitrogen atom, nitrogen-containing and non-nitrogen-containing BPs differ in the mechanism of action exerted on osteoclasts $(13,14)$. Various BPs demonstrate different relative potencies and affinities for bone. The more recent nitrogen-containing BPs, such as zolendronic acid (ZA), are the most potent inhibitors of bone resorption $(13,15)$. ZA is an agent that is administered intravenously, and patients receiving BPs intravenously are at a high risk of developing BP-related osteonecrosis of the jaws (BRONJ) (16).

Different definitions make it challenging to reach conclusions on the incidence of BRONJ, which has been reported to be $<10 \%$ in various studies, and the associated risk factors (17-19). The present study reports a single-centre retrospective study that was performed to determine the frequency, risk factors and clinical presentation of BRONJ, and to also determine the progression of malignancy in patients with cancer treated by ZA once BRONJ develops.

\section{Materials and methods}

Study design. The present study reports a cross-sectional retrospective analysis of all individuals that were treated with a ZA and were diagnosed with BRONJ at Cukurova University Medical School (Adana, Turkey) between January 2008 and December 2012. The control group was randomly selected from the hemato-oncological patients at the same medical centre that were treated with ZA during the same period. All patients in the BRONJ and control groups were examined by the same oral and maxillofacial surgeon, to confirm or reject the diagnosis of BRONJ.

For the patient inclusion criteria, only patients with malignancy that were treated with $4 \mathrm{mg}$ of ZA once every 3 or 6 weeks due to bone metastasis were included. The diagnosis of BRONJ was performed according to the 2009 update of the American Association of Oral and Maxillofacial Surgeons 
position paper (20), specifically exposed bone in the maxillofacial region that had persisted for $>8$ weeks. Patients with head and neck neoplasms and those who received radiotherapy to the head and neck region were excluded.

From medical records, interviews with patients, and questionnaires completed by patients, data were retrieved regarding the age and gender of patients, BP treatment, indication for treatment, drug administered, cumulative dose of agent, duration of treatment, concomitant steroid use, diabetes status, administration of dental treatment, smoking, use of angiogenic agents and state of disease during the development BRONJ in the patients. Written informed consent was obtained from all patients.

Statistical analysis. Statistical analyses in the present study were performed using the Number Cruncher Statistical System 2007 statistical software (NCSS, LLC, Kaysville, UT, USA). To analyze the data, in addition to the descriptive statistical methods of the mean and standard deviation, the independent $t$-test was used for the comparison of dual groups, and $\chi^{2}$ and Fisher's exact tests were used for the comparison of qualitative data.

\section{Results}

Comparison between patients with and without BRONJ. A total of 24 patients that were administered with ZA were diagnosed with BRONJ between January 2008 and December 2012. Examinations performed by the same oral medicine specialist confirmed the diagnoses of BRONJ in all patients in the BRONJ group, and excluded BRONJ in all 20 patients in the control group. The gender distribution in the patients with BRONJ was 11 males and 13 females, and the control group consisted of 8 males and 12 females. In the patients with BRONJ, 37\% $(\mathrm{n}=9)$ possessed ONJ of the mandibular, 20\% $(n=5)$ possessed ONJ of the maxilla, 20\% $(n=5)$ demonstrated multiple location involvement and $6 \%(\mathrm{n}=5)$ possessed $\mathrm{ONJ}$ of unknown location. Out of the 24 patients with BRONJ and the 20 without BRONJ, the indications for ZA treatment consisted of breast cancer in 8 patients (33\%), MM in 8 patients (33\%), prostate cancer in 6 patients $(25 \%)$ and other cancers in 2 patients (8\%), comprising colon cancer and renal cell carcinoma (Table I). The median usage time of ZA was calculated to be 36 months (range, 12-84 months) in the BRONJ group and 27 months (range, 7-65 months) in the control group. No statistically significant difference was observed in the gender distribution of the BRONJ and control groups $(\mathrm{P}=0.697)$ or the diagnosis distribution of the BRONJ and control groups $(\mathrm{P}=0.0090)$.

The median age of the patients with BRONJ was significantly higher than that of the control group $(\mathrm{P}=0.049)$. The cumulative dose medians of the BRONJ group were found to be significantly higher compared with those of the control group ( $\mathrm{P}=0.037$; Table II). The median cumulative dose in the control group was found to be $88 \mathrm{mg}$ (range, $36-120 \mathrm{mg}$ ), while in the BRONJ group the median cumulative dose was $126 \mathrm{mg}$ (range, 72-168 mg).

In the BRONJ group, 13 out of 21 patients (61.9\%) underwent dental treatment for their recent medical condition compared with 4 out of 18 patients (22.2\%) in the control group, which
Table I. Characteristics of patients in the BRONJ and Control groups.

\begin{tabular}{|c|c|c|c|}
\hline & & up & \\
\hline & Control, n (\%) & BRONJ, n (\%) & P-value \\
\hline Gender & & & \\
\hline Female & $12(60.00)$ & $13(54.17)$ & 0.697 \\
\hline Male & $8(40.00)$ & $11(45.83)$ & \\
\hline Diagnosis & & & \\
\hline Prostate & $0(0.00)$ & $6(25.00)$ & 0.090 \\
\hline Breast & $9(45.00)$ & $8(33.33)$ & \\
\hline MM & $7(35.00)$ & $8(33.33)$ & \\
\hline Other & $4(20.00)$ & $2(8.33)$ & \\
\hline $\begin{array}{l}\text { Dental } \\
\text { treatment }\end{array}$ & & & \\
\hline No & 14 (77.78) & $8(38.10)$ & 0.013 \\
\hline Yes & $4(22.22)$ & $13(61.90)$ & \\
\hline DM & & & \\
\hline No & $18(94.74)$ & $19(95.00)$ & 0.970 \\
\hline Yes & $1(5.26)$ & $1(5.00)$ & \\
\hline Use of ste & & & \\
\hline No & $13(68.42)$ & $17(70.83)$ & 0.864 \\
\hline Yes & $6(31.58)$ & 7 (29.17) & \\
\hline Smoker & & & \\
\hline No & $12(75.00)$ & $14(77.78)$ & 0.849 \\
\hline Yes & $4(25.00)$ & $4(22.22)$ & \\
\hline Angiogen & & & \\
\hline No & $14(73.68)$ & $14(70.00)$ & 0.798 \\
\hline Yes & $5(26.32)$ & $6(30.00)$ & \\
\hline State of $d$ & & & \\
\hline $\mathrm{Bad}$ & $11(64.71)$ & $4(26.67)$ & 0.031 \\
\hline Good & $6(35.29)$ & $11(73.33)$ & \\
\hline
\end{tabular}

BRONJ, Bisphosphonate-related osteonecrosis of the jaws; MM, multiple myeloma; DM, diabetes mellitus.

was a statistically significant difference $(\mathrm{P}=0.013)$. When the incidences of diabetes mellitus (DM), smoking and steroid use were compared with those of the control group, the difference was not significant $(\mathrm{P}>0.05)$.

The percentage use of anti-angiogenic agents was $25 \%$ $(n=6)$ in the BRONJ group, with thalidomide being used by 5 patients and bevacizumab being used by 1 patient, while the percentage use was $25 \%(n=5 / 20)$ in the control group, with thalidomide being used by four patients and bevacizumab being used by one patient. No significant difference was observed between the control group and the patients with BRONJ in the usage of anti-angiogenic agents $(\mathrm{P}=0.798$; Table II).

In terms of the progression of the main malignancy during jaw osteonecrosis (ONJ) development, the results were $11 / 24$ (45.8\%) patients with BRONJ, and 6/20 (30\%) control individuals, which demonstrated a significant difference $(\mathrm{P}=0.03)$. 
Table II. BRONJ and control group data according to age and cumulative medication dosage.

\begin{tabular}{lccc}
\hline Characteristic & Control group & BRONJ group & P-value \\
\hline Age, years & $54.3 \pm 7.83$ & $60.17 \pm 11.07$ & \\
& $52(48.25-60.75)$ & $60.5(52.5-68.75)$ & 0.049 \\
Z, months & $26.37 \pm 16.17$ & $35.45 \pm 18.69$ & \\
& $22(16-40)$ & $36(22.5-42.5)$ & 0.094 \\
Cumulative & $94.32 \pm 65.31$ & $135.45 \pm 76.35$ & \\
dose, mg & $88(36-120)$ & $126(72-168)$ & 0.037 \\
\hline
\end{tabular}

Data are expressed at the mean \pm standard deviation. BRONJ, bisphosphonate-related osteonecrosis of the jaws; Z, time of using zoledronic acid.

\section{Discussion}

BRONJ is a side-effect of BP therapy that is a relatively rare complication, but may have a notable impact on the quality of life of the affected patients. At present, the risk factors of BRONJ have been researched in numerous studies $(15,21,22)$.

The present study determined that the frequency of BRONJ reached $3.6 \%$ in patients diagnosed with a malignancy that was treated using intravenous administration of ZA. In the literature, the rate of $\mathrm{ONJ}$ development in patients with malignancies treated using intravenous BP administration has been reported as 1.2, 3.1 and 5\% in different studies $(18,21-25)$. It was hypothesized that other factors such as the usage period of the medication, patient diagnoses, presence of chronic disease and presence of other medication usage may have had an effect on the difference in these rates (23). Also, the differences in ethnicity and particularly in dental hygiene habits affected the risk of BRONJ $(15,21-24)$.

In the present study, $33 \%$ of the patients with ONJ were suffering from breast cancer $(\mathrm{n}=8), 33 \%(\mathrm{n}=8)$ from $\mathrm{MM}$, $25 \%(n=6)$ from prostate cancer and $8 \%(n=2)$ from other cancers, consisting of colon cancer and renal cell carcinoma. No significant association was detected between the diagnosed malignancy and the development of BRONJ $(\mathrm{P}=0.09)$. In certain previous studies, no association was found between the diagnosis and BRONJ, while in other studies it was identified that BRONJ was encountered at a higher rate in patients with $\mathrm{MM}$ in comparison to the patients with breast cancer (15,21-25).

While age was not assessed as a risk factor in the present study, the development of BRONJ occurred in patients with a mean age of 60.5 (range, 52.5-68.75), which was significantly different $(\mathrm{P}=0.04)$ from the age of the control individuals. This may be associated with the reduction in the metabolism of the medication and insufficient mouth care and hygiene in patients of advanced age.

The assessment of the association between the development of BRONJ in patients receiving ZA and the cumulative dose revealed that the cumulative dose of ZA was significantly higher in patients with BRONJ ( $\mathrm{P}=0.03)$. The median cumulative dose was determined to be $88 \mathrm{mg}$ (range, $36-120 \mathrm{mg}$ ) in the control group and $126 \mathrm{mg}$ (range, $72-168 \mathrm{mg}$ ) in the
BRONJ group. The time of exposure to ZA was determined to be 36 months in the BRONJ group and 27 months in the control group. In previous studies, the time of exposure to medication was identified as a risk factor in BRONJ development $(15,21-23)$. However, an optimal treatment time or dose was not clearly suggested with regard to these patients. In another study, no association was determined between the cumulative BP dose and the risk of BRONJ (26).

In the present study, the risk of BRONJ development was found to be significantly higher in patients that underwent tooth extraction while using ZA $(\mathrm{P}=0.01)$. Surgical dental procedures have also been researched in previous studies, and the status of surgical dental procedures as an important risk factor for BRONJ development has been demonstrated (27).

The presence of DM and the incidence of smoking were researched in previous studies as risk factors for the development of BRONJ $(23,28)$. In the present study, the association between DM and smoking and the risk of BRONJ was assessed in the BRONJ and control groups, and the results were not significantly different $(\mathrm{P}=0.09$ and $\mathrm{P}=0.08$, respectively). When the previous studies were considered, evaluating DM and smoking according to the time of exposure to smoking and the time since DM diagnosis and the state of DM regulation was considered to be an improved method for the evaluation of their effects in BRONJ development.

It is also critical to identify the involvement of steroid usage in this rare but important complication of $\mathrm{ZA}$, since a notable number of patients using ZA have MM, and steroids constitute a component of the treatment for MM. Previous studies have identified steroid usage as a risk factor in BRONJ development $(26,29)$. However, in the present study, steroid usage was not monitored as a risk factor $(\mathrm{P}=0.8)$.

The association between an increased risk of BRONJ and the administration of anti-angiogenic agents or other BPs has been investigated in a number of small scale studies (23,30-32). There is a previous study that specifies the use of sunitinib and $\mathrm{ZA}$ as a predisposing factor to the development of BRONJ (30), and there are studies that report bevacizumab to be a risk factor (31-33). However, there are also studies stating that bevacizumab is not a risk factor $(23,34)$. No patients were co-administered with sunitinib and ZA in the present study, but there were patients that used bevacizumab and ZA together. The association of between bevacizumab and ZA and the development of BRONJ was not determined to be statistically significant $(\mathrm{P}=0.7)$.

In the present study, the course of disease was also investigated during the period of BRONJ development, as was the association between ONJ development and the state of malignancy. The state of disease in patients with ONJ was monitored, and was found to be improved during ZA usage, with a statistically significant association $(\mathrm{P}=0.031)$. The most likely cause of the situation may be associated with the long-term usage of ZA. The antitumour effect of the medication may have slowed or stopped the progress of malignancy, due to the long-term usage of ZA. However, BRONJ was encountered more frequently in these patients.

According to the results of the present study, advanced age, the application of dental treatment application and cumulative dose of ZA (median, $126 \mathrm{mg}$ ), were found to be risk factors for BRONJ development. Determination of ZA usage and net 
cumulative dosage in BRONJ development may be presented through a joint calculation to be carried out in the light of other studies that need to be performed. Also, the state of malign disease was determined to be improved during BRONJ development when compared with the patients that did not develop BRONJ. BRONJ development may therefore be a factor during ZA usage, particularly for patients with advanced age. In addition, more frequent and regular dental treatment should be administered to patients with ONJ development, and cooperation between patients, doctors and dentists is required for the treatment of these patients.

\section{References}

1. Black DM, Cummings SR, Karpf DB, et al: Randomised trial of effect of alendronate on risk of fracture in women with existing vertebral fractures. Fracture Intervention Trial Research Group. Lancet 348: 1535-1541, 1996.

2. Berenson JR, Hillner BE, Kyle RA, et al; American Society of Clinical Oncology Bisphosphonates Expert Panel: American Society of Clinical Oncology clinical practice guidelines: The role of bisphosphonates in multiple myeloma. J Clin Oncol 20: 3719-3736, 2002.

3. Fleisch H: Bisphosphonates: Mechanisms of action. Endocr Rev 19: 80-100, 1998.

4. Russell RG, Croucher PI and Rogers MJ: Bisphosphonates: Pharmacology, mechanisms of action and clinical uses Osteoporos Int 9 (Suppl 2): S66-S80, 1999.

5. Löwik CW, van der Pluijm G, van der Wee-Pals LJ, et al: Migration and phenotypic transformation of osteoclast precursors into mature osteoclasts: the effect of a bisphosphonate. J Bone Miner Res 3: 185-192, 1988.

6. Piper K, Boyde A and Jones SJ: The effect of 3-amino-1-hydroxypropylidene-1,1-bisphosphonate (ADP) on the resorptive function of osteoclasts of known nuclear number. Calcif Tissue Int 54: 56-61, 1994.

7. Hughes DE, Wright KR, Uy HL, et al: Bisphosphonates promote apoptosis in murine osteoclasts in vitro and in vivo. J Bone Miner Res 10: 1478-1487, 1995.

8. Reszka AA, Halasy-Nagy JM, Masarachia PJ, et al: Bisphosphonates act directly on the osteoclast to induce caspase cleavage of mst1 kinase during apoptosis. A link between inhibition of the mevalonate pathway and regulation of an apoptosis-promoting kinase. J Biol Chem 274: 34967-34973, 1999.

9. Diel IJ, Solomayer EF and Bastert G: Bisphosphonates and the prevention of metastasis: first evidences from preclinical and clinical studies. Cancer 88: 3080-3088, 2000.

10. Pietschmann P, Stohlawetz P, Brosch S, et al: The effect of alendronate on cytokine production, adhesion molecule expression and transendothelial migration of human peripheral blood mononuclear cells. Calcif Tissue Int 63: 325-330, 1998.

11. Ledoux D, Hamma-Kourbali Y, Di Benedetto M, et al: A new dimethyl ester bisphosphonate inhibits angiogenesis and growth of human epidermoid carcinoma xenograft in nude mice. Anticancer Drugs 17: 479-485, 2006.

12. Rogers MJ, Watts DJ and Russell RG: Overview of bisphosphonates. Cancer 80 (Suppl): 1652-1660, 1997.

13. Boonyapakorn T, Schirmer I, Reichart PA, et al: Bisphosphonate-induced osteonecrosis of the jaws: Prospective study of 80 patients with multiple myeloma and other malignancies. Oral Oncol 44: 857-869, 2008.

14. Green J and Clézardin P: The molecular basis of bisphosphonate activity: a preclinical perspective. Semin Oncol 37 (Suppl 1): S3-S11, 2010.

15. Marx RE: Pamidronate (Aredia) and zoledronate (Zometa) induced avascular necrosis of the jaws: A growing epidemic. J Oral Maxillofac Surg 61: 1115-1117, 2003.

16. Janovská Z: Bisphosphonate-related osteonecrosis of the jaws. A severe side effect of bisphosphonate therapy. Acta Medica (Hradec Kralove) 55: 111-115, 2012.
17. Ferlito S, Puzzo S, Palermo F and Verzì P: Treatment of bisphosphonate-related osteonecrosis of the jaws: presentation of a protocol and an observational longitudinal study of an Italian series of cases. Br J Oral Maxillofac Surg 50: 425-429, 2012.

18. McLeod NM, Brennan PA and Ruggiero SL: Bisphosphonate osteonecrosis of the jaw: A historical and contemporary review. Surgeon 10: 36-42, 2012.

19. Tubiana-Hulin M, Spielmann M, Roux C, et al: Physiopathology and management of osteonecrosis of the jaws related to bisphosphonate therapy for malignant bone lesions. A French expert panel analysis. Crit Rev Oncol Hematol 71: 12-21, 2009.

20. Ruggiero SL, Dodson TB, Assael LA, et al; Task Force on Bisphosphonate-Related Osteonecrosis of the Jaws; American Association of Oral and Maxillofacial Surgeons. American Association of Oral and Maxillofacial Surgeons position paper on bisphosphonate-related osteonecrosis of the jaw - 2009 update. Aust Endod J 35: 119-130, 2009.

21. Hoff AO, Toth BB, Altundag K, et al: Frequency and risk factors associated with osteonecrosis of the jaw in cancer patients treated with intravenous bisphosphonates. J Bone Miner Res 23: 826-836, 2008.

22. Bamias A, Kastritis E, Bamia C, et al: Osteonecrosis of the jaw in cancer after treatment with bisphosphonates: Incidence and risk factors. J Clin Oncol 23: 8580-8587, 2005.

23. Thumbigere-Math V, Tu L, Huckabay S, et al: A retrospective study evaluating frequency and risk factors of osteonecrosis of the jaw in 576 cancer patients receiving intravenous bisphosphonates. Am J Clin Oncol 35: 386-392, 2012.

24. Brufsky AM, Sereika SM, Mathew A, et al: Long-term treatment with intravenous bisphosphonates in metastatic breast cancer: A retrospective study. Breast J 19: 504-511, 2013.

25. Vahtsevanos K, Kyrgidis A, Verrou E, et al: Longitudinal cohort study of risk factors in cancer patients of bisphosphonate-related osteonecrosis of the jaw. J Clin Oncol 27: 5356-5362, 2009.

26. Kos M, Kuebler JF, Luczak $K$ and Engelke W: Bisphosphonate-related osteonecrosis of the jaws: A review of 34 cases and evaluation of risk. J Craniomaxillofac Surg 38: 255-259, 2010.

27. Vescovi P, Campisi G, Fusco V, et al: Surgery-triggered and non-surgery-triggered bisphosphonate-related osteonecrosis of the jaws (BRONJ): A retrospective analysis of 567 cases in an Italian multicentre study. Oral Oncol 47: 191-194, 2011.

28. Molcho S, Peer A, Berg T, et al: Diabetes microvascular disease and the risk for bisphosphonate-related osteonecrosis of the jaw: a single centre study. J Clin Endocrinol Metab 98: E1807-E1812, 2013.

29. Wessel JH, Dodson TB and Zavras AI: Zoledronate, smoking and obesity are strong risk factors for osteonecrosis of the jaw: A case-control study. J Oral Maxillofac Surg 66: 625-631, 2008.

30. Smidt-Hansen T, Folkmar TB, Fode K, et al: Combination of zoledronic acid and targeted therapy is active but may induce osteonecrosis of the jaw in patients with metastatic renal cell carcinoma. J Oral Maxillofac Surg 71: 1532-1540, 2013.

31. Lescaille G, Coudert AE, Baaroun V, et al: Clinical study evaluating the effect of bevacizumab on the severity of zoledronic acid-related osteonecrosis of the jaw in cancer patients. Bone 58: 103-107, 2014.

32. Fusco V, Porta C, Saia G, et al: Osteonecrosis of the jaw in patients with metastatic renal cell cancer treated with bisphosphonates and targeted agents: Results of an Italian multicenter study and review of the literature. Clin Genitourin Cancer: Dec 9, 2014 (Epub ahead of print).

33. McKay RR, Lin X, Perkins JJ, et al: Prognostic significance of bone metastases and bisphosphonate therapy in patients with renal cell carcinoma. Eur Urol 66: 502-509, 2014.

34. McArthur HL, Estilo C, Huryn J, Williams T, Fornier M, Traina TA, Howard J, Hudis CA and Dickler MN: Osteonecrosis of the jaw (ONJ) among intravenous (IV) bisphosphonate- and/or bevacizumab-treated patients (pts) at Memorial Sloan-Kettering Cancer Center (MSKCC). J Clin Oncol 26: 9588, 2008. 